\title{
Collusion and Group Lending with Adverse Selection*
}

\author{
Jea -Jacques Laffo $t^{\dagger}$
}

February 17, 2000

${ }^{*}$ I thank Abhijit Banerjee, Tim Besley, Oliver Hart, Guido Friebel, Patrick Rey, Ilya Segal and Jean Tirole for helpful comments.

${ }^{\dagger}$ IDEI, ARQADE, GREMAQ, Université des Sciences Sociales de Toulouse, Place Anatole-France, 31042 Toulouse Cedex, France. 


\begin{abstract}
In an environment with correlated returns, this paper characterizes optimal lending contracts when the bank faces adverse selection and borrowers have limited liability. Group lending contracts are shown to be dominated by revelation mechanisms which do not use the ex post observability of the partners' performances. However, when collusion between borrowers under complete information is allowed, group lending contracts are optimal in the class of simple revelation mechanisms (which elicit only the borrower's own private information) and remain useful with extended revelation mechanisms.
\end{abstract}

JEL Classification: D8, G2, O12, O17.

Keywords: Group lending, adverse selection, collusion, development. 


\section{Introduction}

The development of group lending through the Grameen Bank and similar institutions has attracted the interest of all those who believe that lending to the poor is a necessary step to exit the vicious circles of underdevelopment. The empirical evaluation of the success of these new ways of lending to entrepreneurs who have no collateral is still subject to debates (see Khandker, Khalily and Khan (1995), Morduch (1997), Pitt and Khandker $(1996))$.

Theorists have proposed various explanations for the new opportunities provided by group lending (see Ghatak and Guinnane (1998) for a review). In this paper we restrict our attention to group lending as an instrument to improve discrimination between entrepreneurs of different types (adverse selection).

Ghatak (2000) and Armendariz and Gollier (2000) have argued that group lending triggers a peer selection effect among entrepreneurs who know each other. For independent types, they show how the knowledge of the types in the group which vary with the different regroupings (for example in a group of two: two good types or two bad types or one good and one bad type) makes discrimination possible. When entrepreneurs do not know each other, with independent types, group lending brings no improvement (Laffont and N'Guessan (1999)).

In this paper we propose a simple model to study the role of group lending in discrimination when collusion between borrowers is possible.

We consider exogenously fixed potential pairs of entrepreneurs who carry projects with correlated returns. Each entrepreneur, when he discovers his type, revises his beliefs about the type of his partner. Nevertheless he does not observe his partner's type. When correlation becomes perfect we have the situation where agents know each other.

We leave aside the issue of endogenous regrouping to focus on two questions: first, what is the relative power of group lending of the Grameen Bank type (called GB contracts), where a successful entrepreneur must contribute to the repayment of his partner if the project of this latter fails, in the class of all possible lending mechanisms? Second, what are the optimal collusion-proof lending contracts and how do the GB contracts perform from the point of view of collusion? 
The model with correlated types is presented in Section 2. Optimal individual lending contracts are characterized in Section 3. The optimal pairing mechanisms which are individually incentive compatible are obtained in Section 4. The place of Grameen Bank (GB) contracts in the class of individually incentive compatible mechanisms is explained in Section 5. Section 6 shows that the GB contracts are in fact optimal when a certain type of group incentive constraints are taken into account. Section 7 considers more general revelation mechanisms and shows that GB contracts remain useful in this context. Section 8 concludes.

\section{The Model}

There is a continuum of pairs of entrepreneurs, each entrepreneur being associated with a good or a bad project. A good (resp. bad) project returns $h$ when it is successful, i.e., with probability $\bar{p}$ (resp. $\underline{p}$ with $\bar{p}>\underline{p}$ ), for one unit of investment. For simplicity we consider only projects of size one.

A pair of entrepreneurs represents a local set of investment opportunities. For simplicity again, we take the case of a group of two entrepreneurs, but, at the cost of more complex notation, it could be a group of any size. However, the size of the group is here exogenous, and we do not raise the issue of the optimal number of entrepreneurs in a group.

Let $p_{i} \in\{\underline{p}, \bar{p}\}$ be the type of entrepreneur $i$ 's project, or, for brevity, the type of the entrepreneur. The types in a pair, $\left(p_{1}, p_{2}\right)$, are jointly distributed according to the distribution function

$$
\begin{aligned}
& \pi_{11}=\operatorname{Pr}\left(p_{1}=\underline{p} \text { and } p_{2}=\underline{p}\right) \\
& \pi_{12}=\operatorname{Pr}\left(p_{1}=\underline{p} \text { and } p_{2}=\bar{p}\right)=\pi_{21}=\operatorname{Pr}\left(p_{1}=\bar{p} \text { and } p_{2}=\underline{p}\right) \\
& \pi_{22}=\operatorname{Pr}\left(p_{1}=\bar{p} \text { and } p_{2}=\bar{p}\right) .
\end{aligned}
$$

Let $\rho=\pi_{11} \pi_{22} \Leftrightarrow \pi_{12} \pi_{21}>0$, a measure of the correlation of types.

Entrepreneurs are risk neutral ${ }^{3}$ and have no wealth. They must borrow to invest and they can only reimburse their loan if their project is successful.

\footnotetext{
${ }^{3}$ To assume risk neutrality may appear inappropriate in this context. However, the assumption of limited liability at zero wealth will play a role similar to risk aversion given that there is no ex post moral hazard dimension in the entrepreneurs' activity.
} 
The lender is a monopolistic bank which has a cost of funds $r$ and which maximizes expected profit. We assume that under complete information all loans are socially valuable because the expected profit from a bad type project is greater than the entrepreneur's opportunity $\operatorname{cost} \underline{p h} \Leftrightarrow r \Leftrightarrow u>0$, when $u$ is the status quo utility level of an entrepreneur outside the relationship with the bank. Efficiency calls for all projects to be financed.

\section{Individual Contracts}

The bank designs individual contracts. An entrepreneur randomly chosen has a probability $\underline{\pi}=\pi_{11}+\pi_{12}\left(\operatorname{resp} . \bar{\pi}=\pi_{21}+\pi_{22}\right)$ of being of type $\underline{p}(\operatorname{resp} . \bar{p})$. From the revelation principle we know that, in order to characterize optimal individual contracts, there is no restriction in considering pairs of contracts

$$
(\underline{P}, \underline{x}),(\bar{P}, \bar{x})
$$

which specify for each type a probability of receiving a loan and a payment if the project is successful. These contracts must satisfy incentive constraints which write: ${ }^{4}$

$$
\begin{aligned}
\underline{P} \underline{p}(h \Leftrightarrow \underline{x}) & \geq \bar{P} \underline{p}(h \Leftrightarrow \bar{x}) \\
\bar{P} \bar{p}(h \Leftrightarrow \bar{x}) & \geq \underline{P} \bar{p}(h \Leftrightarrow \underline{x}) .
\end{aligned}
$$

Participation constraints are:

$$
\begin{aligned}
& \underline{P} \underline{p}(h \Leftrightarrow \underline{x}) \geq u \\
& \bar{P} \bar{p}(h \Leftrightarrow \bar{x}) \geq u .
\end{aligned}
$$

The bank maximizes its expected profit

$$
\underline{\pi P}(\underline{p} \underline{x} \Leftrightarrow r)+(1 \Leftrightarrow \underline{\pi}) \bar{P}(\bar{p} \bar{x} \Leftrightarrow r)
$$

subject to $(1),(2),(3),(4)$.

The bank has two possible strategies. Either to offer contracts which are accepted by both types of entrepreneurs (Regime 1), or to offer a contract which is only accepted by a good type (Regime 2). We obtain immediately (see Appendix 1):

\footnotetext{
${ }^{4}$ For simplicity we assume that borrowers loose their outside opportunity $u$ when they apply for the loan. We obtain similar results if we define utility, say for type $\underline{p}$, as $\underline{P} \underline{p}(h-\underline{x})+(1-\underline{P}) u$.
} 


\section{Proposition 1 If}

$$
\underline{\pi}(\underline{p} h \Leftrightarrow r \Leftrightarrow u)>\bar{\pi} \frac{\bar{p} \Leftrightarrow \underline{\underline{p}}}{\underline{p}} u,
$$

Regime 1 holds. The bank offers a pooling contract which gives a loan to all entrepreneurs for a reimbursement $x=h \Leftrightarrow \underline{\underline{\underline{u}}}$ when the project is successful.

If

$$
\underline{\pi}(\underline{p} h \Leftrightarrow r \Leftrightarrow u)<\bar{\pi} \frac{\bar{p} \Leftrightarrow \underline{p}}{\underline{p}} u
$$

Regime 2 holds. The bank offers a contract accepted only by type $\bar{p}$ entrepreneurs with a reimbursement $x=h \Leftrightarrow \frac{u}{\bar{p}}$ when the project is successful.

In Regime 1, an entrepreneur of type $\bar{p}$ has an expected rent

$$
\bar{p}\left(h \Leftrightarrow\left(h \Leftrightarrow \frac{u}{\underline{p}}\right)\right) \Leftrightarrow u=\frac{\bar{p} \Leftrightarrow \underline{p}}{\underline{p}} \cdot u .
$$

Regime 1 is preferred by the bank if the expected profit to be realized with type $\underline{p}$ entrepreneurs, $\underline{\pi}(\underline{p} h \Leftrightarrow r \Leftrightarrow u)$, exceeds the expected rent,

$$
\bar{\pi} \cdot\left(\frac{\bar{p} \Leftrightarrow \underline{p}}{\underline{p}}\right) u
$$

that must be given up in Regime 1 to type $\bar{p}$ (in contrast to Regime 2 where no rent need to be given to these entrepreneurs), because of the presence of type $\underline{p}$.

In Regime 1 the allocation of loans is efficient, and the good type entrepreneurs are able to obtain a rent despite the monopolistic structure of banking. In Regime 2 the allocation of loans is inefficient since the valuable projects of type $\underline{p}$ are not financed, but the good type entrepreneurs obtain no rent.

\section{Pairing Contracts}

The bank considers now the natural groups of entrepreneurs which are the pairs of entrepreneurs with correlated projects and exploits the fact that the structure of correlation is common knowledge. For notational convenience let us refer to type $\underline{p}$ (resp. $\bar{p}$ ) as type 1 (resp. 2). From the revelation principle again, there is no loss of generality in restricting the offer of contracts to two four-uples, $\left(x_{11}, x_{12}, x_{21}, x_{22}\right),\left(y_{11}, y_{12}, y_{21}, y_{22}\right)$, where $x_{i j}$ is the repayment of an entrepreneur who has announced that he is of type $i$ when his partner 
has announced that he is of type $j$, and when both have succeeded; similarly $y_{i j}$ is the repayment of a successful entrepreneur when his partner has not succeeded, and with the same announcements. ${ }^{5}$

The participation and incentive constraints of type $\underline{p}$ and $\bar{p}$ are respectively (see Appendix 2):

$$
\begin{gathered}
\pi_{11}\left(h \Leftrightarrow \underline{p} x_{11} \Leftrightarrow(1 \Leftrightarrow \underline{p}) y_{11}\right)+\pi_{12}\left(h \Leftrightarrow \bar{p} x_{12} \Leftrightarrow(1 \Leftrightarrow \bar{p}) y_{12}\right) \geq \frac{u}{\underline{p}}\left(\pi_{11}+\pi_{12}\right) \\
\pi_{21}\left(h \Leftrightarrow \underline{p} x_{21} \Leftrightarrow(1 \Leftrightarrow \underline{p}) y_{21}\right)+\pi_{22}\left(h \Leftrightarrow \bar{p} x_{22} \Leftrightarrow(1 \Leftrightarrow \bar{p}) y_{22}\right) \geq \frac{u}{\bar{p}}\left(\pi_{21}+\pi_{22}\right) \\
\pi_{11}\left(h \Leftrightarrow \underline{p} x_{11} \Leftrightarrow(1 \Leftrightarrow \underline{p}) y_{11}\right)+\pi_{12}\left(h \Leftrightarrow \bar{p} x_{12} \Leftrightarrow(1 \Leftrightarrow \bar{p}) y_{12}\right) \\
\geq \pi_{11}\left(h \Leftrightarrow \underline{p} x_{21} \Leftrightarrow(1 \Leftrightarrow \underline{p}) y_{21}\right)+\pi_{12}\left(h \Leftrightarrow \bar{p} x_{22} \Leftrightarrow(1 \Leftrightarrow \bar{p}) y_{22}\right) \\
\pi_{21}\left(h \Leftrightarrow \underline{p} x_{21} \Leftrightarrow(1 \Leftrightarrow \underline{p}) y_{21}\right)+\pi_{22}\left(h \Leftrightarrow \bar{p} x_{22} \Leftrightarrow(1 \Leftrightarrow \bar{p}) y_{22}\right) \\
\geq \pi_{21}\left(h \Leftrightarrow \underline{p} x_{11} \Leftrightarrow(1 \Leftrightarrow \underline{p}) y_{11}\right)+\pi_{22}\left(h \Leftrightarrow \bar{p} x_{12} \Leftrightarrow(1 \Leftrightarrow \bar{p}) y_{12}\right) .
\end{gathered}
$$

Furthermore, we have the wealth constraints:

$$
x_{i j} \leq h \quad ; \quad y_{i j} \leq h \quad \text { for all } i, j
$$

The bank's expected profits are, for a pair of entrepreneurs:

$$
\begin{gathered}
\pi_{11}\left[2 \underline{p}^{2} x_{11}+2 \underline{p}(1 \Leftrightarrow \underline{p}) y_{11}\right]+2 \pi_{12}\left[\underline{p} \bar{p}\left(x_{12}+x_{21}\right)+\underline{p}(1 \Leftrightarrow \bar{p}) y_{12}+(1 \Leftrightarrow \underline{p}) \bar{p} y_{21}\right] \\
+\pi_{22}\left[2 \bar{p}^{2} x_{22}+2 \bar{p}(1 \Leftrightarrow \bar{p}) y_{21}\right] \Leftrightarrow 2 r .
\end{gathered}
$$

The average profit for the continuum of entrepreneurs is:

$$
\pi_{11} \underline{p}\left[\underline{p} x_{11}+(1 \Leftrightarrow \underline{p}) y_{11}\right)+\pi_{12}\left[\underline{p}\left(\bar{p} x_{12}+(1 \Leftrightarrow \bar{p}) y_{12}\right)+\bar{p}\left(\underline{p} x_{21}+(1 \Leftrightarrow \underline{p}) y_{21}\right)\right]
$$

\footnotetext{
${ }^{5}$ We assume that all entrepreneurs of a given type receive or do not receive a loan. This can be proved as in Section 3. We will also focus without loss of generality on symmetric solutions.
} 


$$
\left.+\pi_{22} \bar{p}\left[\bar{p} x_{22}+(1 \Leftrightarrow \bar{p}) y_{22}\right)\right] \Leftrightarrow r
$$

We note that, both in the objective function of the bank and in the constraints, the entrepreneurs' payments enter only through the expected terms $X_{11}=\underline{p} x_{11}+(1 \Leftrightarrow \underline{p}) y_{11}$, $X_{12}=\bar{p} x_{12}+(1 \Leftrightarrow \bar{p}) y_{12}, X_{21}=\underline{p} x_{21}+(1 \Leftrightarrow \underline{p}) y_{21}, X_{22}=\bar{p} x_{22}+(1 \Leftrightarrow \bar{p}) y_{22}$. The wealth constraints are less constraining when $x_{i j}=y_{i j}$ for all $i, j$. So, we can rewrite the bank's program as:

$$
\begin{aligned}
& \max _{\left(X_{i j}\right)} \pi_{11} \underline{p} X_{11}+\pi_{12}\left(\underline{p} X_{12}+\bar{p} X_{21}\right)+\pi_{22} \bar{p} X_{22} \Leftrightarrow r \\
& i, j=1,2
\end{aligned}
$$

s.t.

$$
\begin{aligned}
\pi_{11}\left(h \Leftrightarrow X_{11}\right)+\pi_{12}\left(h \Leftrightarrow X_{12}\right) & \geq \frac{u}{\frac{p}{u}}\left(\pi_{11}+\pi_{12}\right) \\
\pi_{21}\left(h \Leftrightarrow X_{21}\right)+\pi_{22}\left(h \Leftrightarrow X_{22}\right) & \geq \frac{\bar{p}}{\bar{p}}\left(\pi_{21}+\pi_{22}\right) \\
\pi_{11}\left(h \Leftrightarrow X_{11}\right)+\pi_{12}\left(h \Leftrightarrow X_{12}\right) & \geq \pi_{11}\left(h \Leftrightarrow X_{21}\right)+\pi_{12}\left(h \Leftrightarrow X_{22}\right) \\
\pi_{21}\left(h \Leftrightarrow X_{21}\right)+\pi_{22}\left(h \Leftrightarrow X_{22}\right) & \geq \pi_{21}\left(h \Leftrightarrow X_{11}\right)+\pi_{22}\left(h \Leftrightarrow X_{12}\right) \\
X_{i j} & \leq h \text { for all } i, j .
\end{aligned}
$$

We first show that, if the correlation of types is high enough, the optimal pairing contracts are efficient and leave no rent to entrepreneurs.

Proposition 2 When the correlation is high enough ( $p_{12}$ small enough) the optimal contracts of the bank are efficient.

\section{Proof: See Appendix 3.}

The logic here is the one of Crémer-McLean (1988). Using the correlation of types, the bank can design rewards and penalties which both induce truthful revelation of types by entrepreneurs and saturate participation constraints. Here, we find $X_{11}=X_{21}<$ $X_{21}=X_{22}$, i.e., the payment of an entrepreneur is independent of his own type and greater when he is paired with a good type. We do not need to condition contracts on the production level of the partner, but only on announcements (and on the agent's production level because of limited wealth). We will call these contracts unconditional revelation contracts. Difficulties may arise from wealth constraints, but this does not occur for a correlation of types high enough. 
To explore the effects of binding wealth constraints we consider a special case of correlation which can be characterized by a single number:

$$
\pi_{11}=\pi_{22}=\frac{1}{2} \Leftrightarrow \varepsilon \quad ; \quad \pi_{12}=\pi_{21}=\varepsilon \quad ; \quad \rho>0 \Leftrightarrow \varepsilon<\frac{1}{4} .
$$

Then, Proposition 2 holds always if the wealth constraints are not binding, i.e. if (see Appendix 3):

$$
\varepsilon<\varepsilon^{*}=\frac{\underline{p}}{2(\bar{p}+\underline{p})} .
$$

Proposition 3 In the special case, the wealth constraint becomes binding for $\varepsilon>\varepsilon^{*}$. Then the solution is

$$
\begin{aligned}
& X_{12}=X_{22}=h \\
& X_{11}=X_{21}=h \Leftrightarrow \frac{u}{\underline{p}} \cdot \frac{\pi_{11}+\pi_{12}}{\pi_{11}} .
\end{aligned}
$$

Then, the good type obtain a rent

$$
R=\left(\frac{\varepsilon}{\frac{1}{2} \Leftrightarrow \varepsilon} \cdot \frac{\bar{p}}{\underline{p}} \Leftrightarrow 1\right) u .
$$

The bank prefers to offer only a contract to the good type with no rent if

$$
\underline{\pi}(\underline{p} h \Leftrightarrow r \Leftrightarrow u)<\bar{\pi} R
$$

Since $\varepsilon<1 / 4$, then

$$
\frac{\varepsilon}{\frac{1}{2} \Leftrightarrow \varepsilon}<1
$$

Comparing with the case of individual contracts, we see that lending to both types (i.e., efficient lending) occurs more often now.

When the correlation is high, it is possible by using two payments (one when the partner announces he is good, one when he announces he his bad) to discriminate between types and extract all the information rents. This is achieved despite the pooling nature of the optimal contract.

When the correlation of types is small, the bank asks the successful entrepreneur to pay his whole gain $h$ when his partner is bad and must give up a rent to the good type because it is not possible to exploit sufficiently the correlation of types. It shows one limit of yardstick competition ${ }^{6}$ especially in developing countries where limited liability constraints are particularly severe.

${ }^{6}$ See Shleifer (1985), Auriol and Laffont (1992). 


\section{Grameen Bank Contracts}

Group lending has been institutionalized by the Grameen Bank in a particular way. The purpose of this section is to put in perspective these contracts with respect to the optimal contracts characterized in Section 4.

A G.B. contract is characterized, for any entrepreneur, by two possible payments when he is successful: $X$ if his partner is also successful and $Y$ if his partner is not successful.

Participation constraints write:

$$
\begin{aligned}
& \pi_{11} \underline{p}[\underline{p}(h \Leftrightarrow X)+(1 \Leftrightarrow \underline{p})(h \Leftrightarrow Y)]+\pi_{12} \underline{p}[\bar{p}(h \Leftrightarrow X)+(1 \Leftrightarrow \bar{p})(h \Leftrightarrow Y)] \geq u\left(\pi_{11}+\pi_{12}\right) \\
& \pi_{21} \bar{p}[\underline{p}(h \Leftrightarrow X)+(1 \Leftrightarrow \underline{p})(h \Leftrightarrow Y)]+\pi_{22} \bar{p}[\bar{p}(h \Leftrightarrow X)+(1 \Leftrightarrow \bar{p})(h \Leftrightarrow Y)] \geq u\left(\pi_{21}+\pi_{22}\right) .
\end{aligned}
$$

Using the notations of Section 4 we observe that:

$$
\begin{aligned}
& X_{11}=X_{21}=\underline{p} X+(1 \Leftrightarrow \underline{p}) Y \\
& X_{12}=X_{22}=\bar{p} X+(1 \Leftrightarrow \bar{p}) Y .
\end{aligned}
$$

Let $\underline{X}^{*}=X_{11}=X_{21}$ and $\bar{X}^{*}=X_{12}=X_{22}$ the payments in the optimal pairing contracts of Section 4. From (19), (20), we can implement those payments with

$$
\begin{array}{r}
X=\frac{(1 \Leftrightarrow \underline{p}) \bar{X}^{*} \Leftrightarrow(1 \Leftrightarrow \bar{p}) \underline{X}^{*}}{\bar{p} \Leftrightarrow \underline{p}} \\
Y=\frac{\bar{p} \underline{X}^{*} \Leftrightarrow \underline{p} \bar{X}^{*}}{\bar{p} \Leftrightarrow \underline{p}} .
\end{array}
$$

It remains to see when the limited liability constraints become binding. In the example $\pi_{11}=\pi_{21}=\frac{1}{2} \Leftrightarrow \varepsilon, \pi_{12}=\pi_{21}=\varepsilon, Y$ is always less than $h$ and

$$
X \leq h \quad \text { iff } \quad \varepsilon<\frac{1}{2}\left(1 \Leftrightarrow \frac{1}{\underline{p}+\bar{p}}\right) .
$$

As expected, this condition is always more stringent than the one obtained for the optimal contract since

$$
\frac{\underline{p}}{2(\bar{p}+\underline{p})}>\frac{1}{2}\left(1 \Leftrightarrow \frac{1}{\underline{p}+\bar{p}}\right) .
$$


When $\varepsilon$ is too large, $X=h$ and

$$
Y=h \Leftrightarrow \frac{u}{\underline{p}} \frac{1}{(1 \Leftrightarrow \underline{p})+2 \varepsilon(\underline{p} \Leftrightarrow \bar{p})}
$$

with a rent for the good type. ${ }^{7}$

Proposition 4 The optimal Grameen Bank contracts are efficient if correlation is high enough but are not always optimal.

A striking feature is that contrary to the practice of Grameen Banks the payments required from a successful entrepreneur are higher when his partner is successful than when he fails. ${ }^{8}$ This is because we have assumed a positive correlation of types. Payments must differ to use the correlation for rent extraction. The positive correlation implies that it is better to extract more in more likely events. To rationalize in our context with adverse selection the practice of Grameen Bank's contracts for which an additional payment is required when a partner fails we need to assume negative correlation of types. ${ }^{9}$

One could of course offer a menu of Grameen Bank's contracts as in Section 4 to induce entrepreneurs to self-select themselves. However, we saw that it is then useless to vary payments with the success or failure of the partner. It is enough to have them vary with the announcement of type.

Next, we take into account the possibility of collusive behavior.

\section{Collusion under Complete Information}

Let us first assume that entrepreneurs may collude when they play the revelation mechanism offered by the bank but after having accepted the offer of the bank. Accordingly, the participation constraints remain the interim individual participation constraints. Furthermore, we assume that entrepreneurs always share their private information after having

\footnotetext{
${ }^{7}$ Of course, loans to only good types occur in similar circumstances as in Section 2.

${ }^{8}$ Note that this feature creates an incentive for an entrepreneur to make his partner's project unsuccessful.

${ }^{9}$ From a technical point of view Grameen Bank contracts use an additional ex post signal correlated with the type of the agent, namely the success or failure of the partner. As in Riordan and Sappington (1988) only limited liability prevents the principal to extract all the agent's surplus.
} 
accepted the bank's contract, and that a ringmaster organizes the collusion. More precisely we have the following timing: ${ }^{10}$

\begin{tabular}{cccccc}
\hline Lntrepreneurs & Bank & Entrepreneurs & Entrepreneurs & An uninformed & $G \circ S$ \\
obtain their & offers & accept or & share & ringmaster & is \\
private & mechanism & reject & information & offers a & played \\
information & $G$ & $G$ & & collusion & contract $S$
\end{tabular}

Because entrepreneurs share information, individual incentive constraints are dominant strategy incentive constraints

$$
\begin{aligned}
& h \Leftrightarrow \underline{p} x_{11} \Leftrightarrow(1 \Leftrightarrow \underline{p}) y_{11} \geq h \Leftrightarrow \underline{p} x_{21} \Leftrightarrow(1 \Leftrightarrow \underline{p}) y_{21} \\
& h \Leftrightarrow \bar{p} x_{12} \Leftrightarrow(1 \Leftrightarrow \bar{p}) y_{12} \geq h \Leftrightarrow \bar{p} x_{22} \Leftrightarrow(1 \Leftrightarrow \bar{p}) y_{22} \\
& h \Leftrightarrow \underline{p} x_{21} \Leftrightarrow(1 \Leftrightarrow \underline{p}) y_{21} \geq h \Leftrightarrow \underline{p} x_{11} \Leftrightarrow(1 \Leftrightarrow \underline{p}) y_{11} \\
& h \Leftrightarrow \bar{p} x_{22} \Leftrightarrow(1 \Leftrightarrow \bar{p}) y_{22} \geq h \Leftrightarrow \bar{p} x_{12} \Leftrightarrow(1 \Leftrightarrow \bar{p}) y_{12} .
\end{aligned}
$$

When internal transfers are available within the coalition, collusion-proof constraints simplify to, for a pair $(1,1)$ :

$$
\begin{gathered}
2\left(h \Leftrightarrow \underline{p} x_{11} \Leftrightarrow(1 \Leftrightarrow \underline{p}) y_{11}\right) \\
\geq 2 h \Leftrightarrow \underline{p} x_{i j} \Leftrightarrow(1 \Leftrightarrow \underline{p}) y_{i j} \Leftrightarrow \underline{p} x_{j i} \Leftrightarrow(1 \Leftrightarrow \underline{p}) y_{j i} \text { for all } i, j,
\end{gathered}
$$

for a pair $(1,2)$ :

$$
\begin{gathered}
\underline{p}\left(h \Leftrightarrow \bar{p} x_{12} \Leftrightarrow(1 \Leftrightarrow \bar{p}) y_{12}\right)+\bar{p}\left(h \Leftrightarrow \underline{p} x_{21} \Leftrightarrow(1 \Leftrightarrow \underline{p}) y_{21}\right) \geq \\
\underline{p}\left(h \Leftrightarrow \bar{p} x_{i j} \Leftrightarrow(1 \Leftrightarrow \bar{p}) y_{i j}\right)+\bar{p}\left(h \Leftrightarrow \underline{p} x_{j i} \Leftrightarrow(1 \Leftrightarrow \underline{p}) y_{j i}\right) \quad \text { for all } i, j,
\end{gathered}
$$

for a pair $(2,2)$ :

$$
2\left(h \Leftrightarrow \bar{p} x_{22} \Leftrightarrow(1 \Leftrightarrow \bar{p}) y_{22}\right)
$$

\footnotetext{
${ }^{10}$ We are not taking into account the financial constraints in the collusive side-contracts proposed by the ringmaster. This exaggerates the threat of collusion, but it is of no relevance for the results we present below. Furthermore, it can be justified if investors have hidden wealth they can use in their side-contracting.
} 


$$
\geq 2 h \Leftrightarrow \bar{p} x_{i j} \Leftrightarrow(1 \Leftrightarrow \bar{p}) y_{i j} \Leftrightarrow \bar{p} x_{j i} \Leftrightarrow(1 \Leftrightarrow \bar{p}) y_{j i} \quad \text { for all } i, j
$$

If we proceed as in Section 4 and do not distinguish payments according to the success or failure of the partner, i.e., $X_{i j}=x_{i j}=y_{i j}$ for all $i, j$, incentive constraints imply

$$
X_{11}=X_{21}=X_{22}=X_{12}
$$

We are then back immediately to the individual contracts of Section 2.

Suppose on the contrary that we keep the flexibility of $x_{i j} \neq y_{i j}$.

Dominant strategy incentive constraints imply

$$
\begin{aligned}
\underline{p} x_{11}+(1 \Leftrightarrow \underline{p}) y_{11} & =\underline{p} x_{21}+(1 \Leftrightarrow \underline{p}) y_{21} \\
\bar{p} x_{22}+(1 \Leftrightarrow \bar{p}) y_{22} & =\bar{p} x_{12}+(1 \Leftrightarrow \bar{p}) y_{12},
\end{aligned}
$$

and the collusion-proof constraints reduce to

$$
\begin{aligned}
\underline{p} x_{11}+(1 \Leftrightarrow \underline{p}) y_{11} \leq \underline{p} x_{12}+(1 \Leftrightarrow \underline{p}) y_{12} \\
\bar{p} x_{22}+(1 \Leftrightarrow \bar{p}) y_{22} \leq \bar{p} x_{21}+(1 \Leftrightarrow \bar{p}) y_{21} \\
\underline{p} x_{21}+(1 \Leftrightarrow \underline{p}) y_{21} \leq \underline{p} x_{22}+(1 \Leftrightarrow \underline{p}) y_{22} \\
\bar{p} x_{12}+(1 \Leftrightarrow \bar{p}) y_{12} \leq \bar{p} x_{11}+(1 \Leftrightarrow \bar{p}) y_{11},
\end{aligned}
$$

with the wealth constraints:

$$
\begin{array}{ll}
x_{i j} \leq h & \text { for all } i, j \\
y_{i j} \leq h & \text { for all } i, j
\end{array}
$$

With the interim participation constraints and the incentive constraints we have eight constraints and also eight variables. Furthermore, we have the wealth constraints.

Imposing

$$
\begin{aligned}
& x_{11}=x_{12}=x_{21}=x_{22}=X \\
& y_{11}=y_{12}=y_{21}=y_{22}=Y
\end{aligned}
$$

enables us to satisfy all individual and coalition incentive constraints, and also to extract all the rents if

$$
\begin{aligned}
& h \Leftrightarrow \frac{\underline{p} \pi_{11}+\bar{p} \pi_{12}}{\pi_{11}+\pi_{12}} X \Leftrightarrow \frac{(1 \Leftrightarrow \underline{p}) \pi_{11}+(1 \Leftrightarrow \bar{p}) \pi_{12}}{\pi_{11}+\pi_{12}} Y=\frac{u}{\underline{p}} \\
& h \Leftrightarrow \frac{\underline{p} \pi_{21}+\bar{p} \pi_{22}}{\pi_{21}+\pi_{22}} X \Leftrightarrow \frac{(1 \Leftrightarrow \underline{p}) \pi_{21}+(1 \Leftrightarrow \bar{p}) \pi_{22}}{\pi_{21}+\pi_{22}} Y=\frac{u}{\bar{p}} .
\end{aligned}
$$


The determinant of this system is $(\underline{p} \Leftrightarrow \bar{p}) \rho$ which is non null as soon as there is some correlation. We can find $X$ and $Y$ which solve the system.

In the case $\pi_{11}=\pi_{22}=\frac{1}{2} \Leftrightarrow \varepsilon, \pi_{12}=\pi_{21}=\varepsilon$, we obtain:

$$
\begin{aligned}
& X=h \Leftrightarrow \frac{u}{\underline{p} \bar{p}}\left[\frac{(1 \Leftrightarrow 2 \varepsilon)(\bar{p}+\underline{p}) \Leftrightarrow 1}{1 \Leftrightarrow 4 \varepsilon}\right] \\
& Y=h \Leftrightarrow \frac{u}{\underline{p} \bar{p}}\left[\frac{(1 \Leftrightarrow 2 \varepsilon)(\bar{p}+\underline{p})}{1 \Leftrightarrow 4 \varepsilon}\right]<h .
\end{aligned}
$$

with

$$
X<h \quad \text { if } \quad \varepsilon<\frac{1}{2}\left(1 \Leftrightarrow \frac{1}{\underline{p}+\bar{p}}\right) .
$$

We obtain (for $\underline{p}+\bar{p}>1)$ :

Proposition 5 The optimal collusion-proof contract is the optimal Grameen Bank contract if correlation is high enough. ${ }^{11}$

Note that the optimal collusion-proof contract is not here what would result from the optimal contract of Section 4 with collusion. Indeed, then they would always claim that they are both bad types (since from Appendix 3 we notice that $X_{12}=X_{22}>X_{11}=X_{21}$ ). They would always pay $X_{11}$ and therefore the pair of good types would have a rent contrary to what is achieved in the Grameen bank contract for a high correlation.

Grameen Bank contracts have been presented in the literature (Ghatak (2000), Armendariz and Gollier (2000)) as useful to allow some discrimination between types. We have shown that their value for discrimination is limited and that, for this purpose, they are dominated by contracts which vary payments as a function of the agent's announcements. However, these latter contracts are not collusion-proof if agents can collude when they play the announcement game. On the contrary, the GB contracts are robust to this type of collusion while still allowing some discrimination. This is achieved by exploiting the correlation of types in the uncertainty on final production.

\footnotetext{
${ }^{11}$ In the limit for $\varepsilon=0$ it seems it is as if the bank was facing a single agent. This is true for incentive constraints, but we still have two participation constraints that the bank can saturate because, by observing the success or failure of both projects, it still has two degrees of freedom.
} 


\section{$7 \quad$ Extended Mechanisms}

We assume now that the mechanism asks from agents the whole vector of types once they have shared their information. Any deviation from the sending of the same messages by the two agents is punished. We are left with the collusion-proof constraints as incentive constraints.

Suppose one does not distinguish payments according to the success or failure of the partner. We call these mechanisms unconditional extended revelation mechanisms. These constraints reduce to

$$
\begin{gathered}
X_{11}=X_{22} \leq \frac{X_{12}+X_{21}}{2} \\
\frac{\underline{p} X_{12}+\bar{p} X_{21}}{2} \leq X_{11}=X_{22} .
\end{gathered}
$$

Proposition 6 Unconditional extended revelation mechanisms cannot be collusion-proof and efficient.

See Appendix 4 for the proof. The intuition of this result is that, for a coalition, we have three types $(\underline{p p}, \underline{p} \bar{p}, \bar{p} \bar{p})$ and three revelant incentive constraints at least, and also a participation constraint for each agent, hence five constraints, and only four degrees of freedom.

The added flexibility of unconditional extended revelation mechanisms is not enough to achieve efficiency, while simple Gramen Bank contract do achieve efficiency when the correlation is high enough. (Proposition 4 remains valid with collusion when extended mechanisms are used.) Using Grameen Bank extended mechanisms will increase the range of parameters for which efficiency is achieved. We can safely conclude that Grameen Bank contracts remain useful to deal with collusion in this extended framework.

We have assumed so far that agents share information with or without collusion. The bank knows that agents will discover the characteristics of both agents and can use extended mechanisms. If instead, the third party only has the technology for sharing information, in a mechanism which does not elicit the fact that agents collude, the bank cannot use extended mechanisms and we must combine the complete information collusion-proof constraints of Section 6 with individual Bayesian incentive constraints. However, the bank could also force communication with the third party by asking both pieces of information 
and use collusion-proof extended mechanisms. Then we are back to this section. With a benevolent third party the two modellings lead to the same results.

Another interesting situation occurs when the sharing of information occurs only with some probability. Then one can argue that extended mechanisms cannot be used if the principal wants to be sure to satisfy the individual rationality constraints. But then the collusion-proof constraints must be written under incomplete information, ${ }^{12}$ leaving open the relevance of collusion under complete information with some probability. However, Grameen Bank will remain in general useful when collusion is an issue despite the fact that unconditional revelation contracts perform better because of the transaction costs of collusion due to asymmetric information.

\section{Conclusion}

We have considered an extremely simple model to make two points. On the one hand, GB contracts are a particular way of practicing a subtle type of discrimination and constitute a powerful tool of rent extraction when types are correlated. However, they are not the optimal such instruments and furthermore they require negative correlation of types to rationalize payments higher when the partner fails. On the other hand, we have shown that GB contracts are interesting to extract rents when collusive behavior is possible.

These results should be robust to more general situations with loans of variable sizes, endogenous grouping. It is obvious for the first result. It relies on the difficulties of enforcing collusion ex post for the second.

We leave for further research a more detailed analysis of the optimal collusion-proof contracts when collusion takes places under asymmetric information with limited liability constraints taking into account in particular in the design of side-contracts.

A necessary next step for the analysis will be to consider dynamic situations. With full commitment of the bank, the emphasis should be on the opportunities of dynamic collusion. Without full commitment, we will have to deal with the additional problems of ratchet effects.

\footnotetext{
${ }^{12}$ See Laffont and Martimort (1999).
} 


\section{Appendix 1: Proof of Proposition 1}

Regime 1: Constraints (1), (2), (3), (4) must be satisfied. (1) and (2) imply $\underline{P}(h \Leftrightarrow \underline{x})=$ $\bar{P}(h \Leftrightarrow \bar{x})$. Since $\bar{p}>\underline{p},(3)$ will be binding and (4) not. Hence:

$$
\underline{x}=h \Leftrightarrow \frac{u}{\underline{p} \underline{P}} \quad ; \quad \bar{x}=h \Leftrightarrow \frac{u}{\underline{p} \bar{P}} .
$$

Substituting these expressions in the bank's expected profit we get:

$$
\underline{\pi P}(\underline{p} h \Leftrightarrow r)+\bar{\pi} \bar{P}(\bar{p} h \Leftrightarrow r) \Leftrightarrow \underline{\pi} u \Leftrightarrow \bar{\pi} \frac{\bar{p}}{\underline{p}}
$$

Since $\underline{p} h \Leftrightarrow r>0$, maximizing with respect to $\underline{P}$ and $\bar{P}$ gives $\underline{P}=\bar{P}=1, \underline{x}=\bar{x}=h \Leftrightarrow \underline{u} \underline{\underline{u}}$ (i.e. a pooling contract), and an expected profit

$$
\underline{\pi}(\underline{p} h \Leftrightarrow r \Leftrightarrow u)+\bar{\pi}\left(\bar{p} h \Leftrightarrow r \Leftrightarrow \frac{\bar{p}}{\underline{p}} u\right) .
$$

Regime 2: The bank offers a loan intended only for type $\bar{p}$. It is constrained only by (4), type $\bar{p}$ 's participation constraint, hence

$$
\bar{x}=h \Leftrightarrow \frac{u}{\bar{p}}
$$

which leaves no rent to type $\bar{p}$ and an expected profit

$$
\bar{\pi}(\bar{p} h \Leftrightarrow r \Leftrightarrow u)
$$

Regime 2 is better than regime 1 if

$$
\bar{\pi}(\bar{p} h \Leftrightarrow r \Leftrightarrow u)>\underline{\pi}(\underline{p} h \Leftrightarrow r \Leftrightarrow u)+\bar{\pi}\left(\bar{p} h \Leftrightarrow r \Leftrightarrow \frac{\bar{p}}{\underline{p}} u\right),
$$

or

$$
\underline{\pi}(\underline{p} h \Leftrightarrow r \Leftrightarrow u)<\bar{\pi} \frac{\bar{p} \Leftrightarrow \underline{p}}{\underline{p}} u .
$$




\section{Appendix 2: Bayesian Incentive and \\ Participation Constraints}

Consider type $\underline{p}$. His posterior probabilities about the type of his partner are:

$$
\frac{\pi_{11}}{\pi_{11}+\pi_{12}} \quad \text { for type } \underline{p}
$$

and

$$
\frac{\pi_{12}}{\pi_{11}+\pi_{12}} \quad \text { for type } \bar{p}
$$

His (Bayesian) participation constraint is:

$$
\begin{gathered}
\frac{\pi_{11}}{\pi_{11}+\pi_{12}} \underline{p}\left(h \Leftrightarrow \underline{p} x_{11} \Leftrightarrow(1 \Leftrightarrow \underline{p}) y_{11}\right) \\
+\frac{\pi_{12}}{\pi_{11}+\pi_{12}} \underline{p}\left(h \Leftrightarrow \bar{p} x_{12} \Leftrightarrow(1 \Leftrightarrow \bar{p}) y_{12}\right) \geq u
\end{gathered}
$$

hence (6) and similarly for (7).

His (Bayesian) incentive constraint is

$$
\begin{aligned}
& \frac{\pi_{11}}{\pi_{11}+\pi_{12}} \underline{p}\left(h \Leftrightarrow \underline{p} x_{11} \Leftrightarrow(1 \Leftrightarrow \underline{p}) y_{11}\right)+\frac{\pi_{12}}{\pi_{11}+\pi_{12}} \underline{p}\left(h \Leftrightarrow \bar{p} x_{12} \Leftrightarrow(1 \Leftrightarrow \bar{p}) y_{12}\right) \\
\geq & \frac{\pi_{11}}{\pi_{11}+\pi_{12}} \underline{p}\left(h \Leftrightarrow \underline{p} x_{21} \Leftrightarrow(1 \Leftrightarrow \underline{p}) y_{21}\right)+\frac{\pi_{12}}{\pi_{11}+\pi_{12}} \underline{p}\left(h \Leftrightarrow \bar{p} x_{22} \Leftrightarrow(1 \Leftrightarrow \bar{p}) y_{22}\right)
\end{aligned}
$$

hence (8) and similarly for (9). 


\section{Appendix 3: Proof of Proposition 2}

Equations (12) to (15) can be rewritten:

$$
\left[\begin{array}{cccc}
\Leftrightarrow \pi_{11} & \Leftrightarrow \pi_{12} & 0 & 0 \\
0 & 0 & \Leftrightarrow \pi_{21} & \Leftrightarrow \pi_{22} \\
\Leftrightarrow \pi_{11} & \Leftrightarrow \pi_{11} & \pi_{11} & \pi_{12} \\
\pi_{21} & \pi_{22} & \Leftrightarrow \pi_{21} & \Leftrightarrow \pi_{22}
\end{array}\right]\left[\begin{array}{c}
X_{11} \\
X_{12} \\
X_{21} \\
X_{22}
\end{array}\right]=\left[\begin{array}{c}
\left(\pi_{11}+\pi_{12}\right)\left(\frac{u}{p} \Leftrightarrow h\right) \\
\left(\pi_{21}+\pi_{22}\right)\left(\frac{u}{\bar{p}} \Leftrightarrow h\right) \\
0 \\
0
\end{array}\right]=\left[\begin{array}{c}
a \\
b \\
0 \\
0
\end{array}\right]
$$

The determinant of this system is $\Delta=\Leftrightarrow p^{2}$.

Solving the system we obtain:

$$
\begin{aligned}
& X_{11}=X_{21}=\frac{b \pi_{12} \Leftrightarrow a \pi_{22}}{\rho}=h \Leftrightarrow \frac{u}{\underline{p}} \Leftrightarrow \frac{u}{\rho} \pi_{12}\left(\pi_{12}+\pi_{22}\right) \cdot \frac{\bar{p} \Leftrightarrow \underline{p}}{\bar{p} \underline{p}} \\
& X_{12}=X_{22}=\frac{a \pi_{21} \Leftrightarrow b \pi_{11}}{\rho}=h \Leftrightarrow \frac{u}{\bar{p}}+\frac{u}{\rho} \pi_{21}\left(\pi_{11}+\pi_{12}\right) \cdot \frac{\bar{p} \Leftrightarrow \underline{p}}{\bar{p} \underline{p}} .
\end{aligned}
$$

These payments satisfy the limited liability constraint if $\pi_{12}$ is close enough to zero. Then, both entrepreneurs are indifferent between lying or telling the truth about their type and no rent is given up. They are rewarded if their partner is good and punished if he is bad.

As $\pi_{12}$ increases we reach the boundary $h$ for $X_{12}$.

For example if $\pi_{11}=\pi_{22}=\frac{1}{2} \Leftrightarrow \varepsilon$ and $\pi_{12}=\pi_{21}=\varepsilon$ the boundary is reached for

$$
\varepsilon^{*}=\frac{\underline{p}}{2(\bar{p}+\underline{p})}
$$

For $\varepsilon>\varepsilon^{*}$, the solution entails $X_{12}=X_{22}=h$, hence the constraints:

$$
\begin{aligned}
\pi_{11}\left(h \Leftrightarrow X_{11}\right) & \geq \frac{u}{\frac{p}{u}}\left(\pi_{11}+\pi_{12}\right) \\
\pi_{21}\left(h \Leftrightarrow X_{21}\right) & \geq \frac{\bar{p}}{\bar{p}}\left(\pi_{21}+\pi_{22}\right) \\
\pi_{11}\left(h \Leftrightarrow X_{11}\right) & \geq \pi_{11}\left(h \Leftrightarrow X_{21}\right) \\
\pi_{21}\left(h \Leftrightarrow X_{21}\right) & \geq \pi_{21}\left(h \Leftrightarrow X_{11}\right) .
\end{aligned}
$$

From (A.3) (A.4), $X_{11}=X_{21}=X$. 
We can expect the bad type's participation constraint to be binding

$$
X=h \Leftrightarrow \frac{u}{\underline{p}} \cdot \frac{\pi_{11}+\pi_{12}}{\pi_{11}}
$$

with an expected rent for the good type:

$$
\left[\frac{\pi_{21}\left(\pi_{11}+\pi_{12}\right)}{\pi_{11}\left(\pi_{21}+\pi_{22}\right)} \frac{\bar{p}}{p} \Leftrightarrow 1\right] u .
$$

In the special case this is positive if $\varepsilon$ is larger than $\varepsilon^{*}$. 


\section{Appendix 4: Proof of Proposition 5}

Let us denote $x=X_{11}=X_{22}$.

To saturate both individual rationality constraints we need

$$
\begin{aligned}
& X_{12}=\Leftrightarrow \frac{\pi_{11}}{\pi_{12}} x+\frac{\pi_{11}+\pi_{12}}{\pi_{12}}\left(h \Leftrightarrow \frac{u}{\underline{p}}\right) \\
& X_{21}=\Leftrightarrow \frac{\pi_{22}}{\pi_{21}} x+\frac{\pi_{21}+\pi_{22}}{\pi_{21}}\left(h \Leftrightarrow \frac{u}{\bar{p}}\right) .
\end{aligned}
$$

The constraint

$$
\frac{X_{12}+X_{21}}{2} \geq X_{22}=X_{11}
$$

writes now

$$
x \leq h \Leftrightarrow\left(\pi_{11}+\pi_{12}\right) \frac{u}{\underline{p}} \Leftrightarrow\left(\pi_{21}+\pi_{22}\right) \frac{u}{\bar{p}} .
$$

The constraint

$$
\frac{\underline{p} X_{12}+\bar{p} X_{21}}{\underline{p}+\bar{p}} \leq X_{11}=X_{22}
$$

writes now

$$
x \geq h \Leftrightarrow \frac{u}{\underline{p}\left(\pi_{11}+\pi_{12}\right)+\bar{p}\left(\pi_{21}+\pi_{22}\right)} .
$$

These inequalities are compatible if

$$
\underline{p}^{2}+\bar{p}^{2}+\underline{p} \bar{p}\left[\frac{\left(\pi_{11}+\pi_{12}\right)^{2}+\left(\pi_{21}+\pi_{22}\right)^{2} \Leftrightarrow 1}{\left(\pi_{11}+\pi_{12}\right)\left(\pi_{22}+\pi_{21}\right)}\right] \leq 0 .
$$

But the left hand side equals

$$
\underline{p}^{2}+\bar{p}^{2} \Leftrightarrow 2 \underline{p} \bar{p}=(\bar{p} \Leftrightarrow \underline{p})^{2}>0 \quad \text { if } \bar{p}>\underline{p} .
$$




\section{REFERENCES}

Armendariz de Aghion, B. and C. Gollier (2000), "Peer Group Formation in an Adverse Selection Model", forthcoming in Economic Journal.

Auriol, E. and J.J. Laffont (1992), "Regulation by Duopoly", Journal of Economics and Management Strategy, 1, 503-533.

Ghatak, M. (2000), "Joint Liability Credit Contracts and the Peer Selection Effect", forthcoming in Economic Journal.

Ghatak, M. and T. Guinnane (1998), "The Economics of Lending with Joint Liability: A Review of Theory and Practice", mimeo, University of Chicago and Yale University.

Khandker, S., B. Khalily and Z. Khan (1995), "Grameen Bank: Performance and Sustainability", World Bank, Discussion Paper 306, Washington D.C.

Laffont, J.J. and D. Martimort (1999), "Mechanism Design with Collusion and Correlation", Econometrica, forthcoming.

Laffont, J.J. and T.T. N'Guessan (1999), "Group Lending with Adverse Selection", to appear in European Economic Review.

Morduch, J. (1997), "The Microfinance Revolution", mimeo, Department of Economics and HIID, Harvard University, Cambridge.

Pitt, M. and S. Khandker (1996), "Household and Intrahousehold Impact of the Grameen Bank and Similar Targeted Credit programs in Bangladesh", World Bank, Discussion Paper 320, Washington D.C.

Riordan, M. and D. Sappington (1988), "Optimal Contracts with Public Ex Post Information", Journal of Economic Theory, 45, 189-199.

Shleifer, A. (1985), "A Theory of Yardstick Competition", Rand Journal of Economics, 16, 319-327. 